\title{
Factorization of Meromorphic Functions in Several Complex Variables
}

\author{
BAO QIN LI AND CHUNG-CHUN YANG
}

\section{Introduction}

Let $F(z)$ be a meromorphic function in the complex plane $\mathbb{C}=\{z|| z \mid<+\infty\}$. $F(z)$ is said to have a factorization with left factor $f$ and right factor $g$ provided

$$
F(z)=f(g(z)),
$$

where $f$ is a meromorphic function in $\mathbb{C}$ and $g$ is an entire function in $\mathbb{C}(g$ may be meromorphic when $f$ is rational). $F(z)$ is said to be prime (pseudoprime, left-prime, and right-prime) if every factorization of form (1.1) implies that either $f$ is bilinear of $g$ is linear (either $f$ is rational or $g$ is a polynomial, $f$ must be bilinear whenever $g$ is transcendental, $g$ must be linear whenever $f$ is transcendental). The first example of prime functions is $F(z)=e^{z}+z$ given by Rosenbloom $[R]$ who investigated the existence and quantitative estimation of the fixed-point of a composite function of two entire functions. He asserted without proof that $e^{z}+z$ is prime. The primeness of this function was first proved by Gross [G1]. Since then there have been several different proofs which have suggested several directions in the development of the theory. While factorization theory of meromorphic functions of one complex variable has been pursued by numerous authors in various asperts and many related topics may have been intensely studied, (c.f. [G1, G2, G3, CY, Y1, Y2]), there is nothing about the theory of several complex variables in the literature.

The main purpose of the present paper is to try to extend and promote the research of factorization theory of meromorphic functions in one complex variable $z$ to several complex variables $Z$ (or $\xi$ ). The first problem we encounter here is how to give a proper definition of the primeness of entire or meromorphic functions of several complex variables. Given a meromorphic function $F(Z)$ :

1991 Mathematics Subject Classification. 32A99, 32A15, 32A22.

Research of the second author is partially supported by an UPGC grant of Hong Kong.

This paper is in final form and no version of it will be submitted for publication elsewhere.

(C) 1993 American Mathematical Society $0271-4132 / 9381.00+\$ .25$ per page 
$\mathbb{C}^{n} \rightarrow \mathbb{P}^{1}:=\mathbb{C} \cup\{\infty\}$, one might consider the following "natural factorization": $F(Z)=f(g)$, where $g: \mathbb{C}^{n} \rightarrow \mathbb{C}^{m}(m \geqslant 1$ a positive integer $)$ is an entire map and $f: \mathbb{C}^{m} \rightarrow \mathbb{P}^{1}$ is a meromorphic function, and define the corresponding concept of primeness, accordingly. However, it is easy to see that such a definition is not compatible with the theory in one variable as is shown by the example $F(z)=$ $e^{z}+z$. In fact, $F(z)$ is prime in $\mathbb{C}$, but $F(z)=: f(g(z))$ with $g=\left(e^{z}, 1+z e^{-z}\right)$, an entire map from $\mathbb{C}$ to $\mathbb{C}^{2}$ and $f=\xi_{1} \xi_{2}$, an entire function from $\mathbb{C}^{2}$ to $\mathbb{C}$. Both of $f$ and $g$ are non-linear. Hence the usual prime function is not prime any more in the above sense. This observation shows that it is reasonable just to consider the factorization of "functions", without any "maps" involved. We thus pose the following general definition for the primeness of a meromorphic function of several complex variables.

Definition 1.1. Let $F(Z)$ be a meromorphic function in $\mathbb{C}^{n} . F(Z)$ is said to have a factorization with left factor $f$ and right factor $g$ provided

$$
F(Z)=f(g(Z)),
$$

where $f$ is a meromorphic function from $\mathbb{C}$ to $\mathbb{P}^{1}$ and $g$ is an entire function from $\mathbb{C}^{n}$ to $\mathbb{C}$ ( $g$ may be a meromorphic function from $\mathbb{C}^{2}$ to $\mathbb{P}^{1}$ when $f$ is a rational function from $\mathbb{C}$ to $\mathbb{P}^{1}$ ). $F(Z)$ is said to be prime in $\mathbb{C}^{n}$ (pseudo-prime, leftprime, and right-prime) if every factorization of form (1.2) implies that either $f$ is bilinear in $\mathbb{C}$ or $g$ is linear in $\mathbb{C}^{\text {n }}$ (either $f$ is rational in $\mathbb{C}$ or $g$ is a polynomial in $\mathbb{C}^{n}, f$ must be bilinear in $\mathbb{Q}$ whenever $g$ is transcendental in $\mathbb{C}^{n}, g$ must be linear in $\mathbb{C}^{n}$ whenever $f$ is transcendental in $\mathbb{C}$ ).

As mentioned above, $F(z)=e^{z}+z$ s the first example ever exhibited to be prime in $\mathbb{C}$. Thus it becomes very natural for us to test whether its "extension" in $\mathbb{C}^{n}$ is prime. That is, can we prove that $F(\xi)=e^{\xi_{1}+\xi_{2}+\cdots+\xi_{n}}+a_{1} \xi_{1}+\cdots+a_{n} \xi_{n}$ $\left(a_{i}\right.$ 's are complex numbers, not all zero is prime in $\mathbb{C}^{n}$ ? An affirmative answer will be given as a direct consequence of our main result Theorem 3.1.

We divide the paper into four parts The first part is the introduction. In Section 2, we recall some basic notations and properties of the growth and divisor of a meromorphic function in $\mathbb{C}^{n}$ which are needed in our proofs. In Section 3, we give a criterion for the primeness of a meromorphic function in $\mathbb{C}^{n}$ (Theorem 3.1) which will enable us to show: as a corollary, that the above function $F(\xi)=$ $e^{\sum_{i=1}^{n} \xi_{i}}+\sum_{i=1}^{n} a_{i} \xi_{i}$ is prime. In the final section, we compare the relation between the primeness of a meromorphi: function $F(\xi)$ in $\mathbb{C}^{n}$ and its restrictions $F\left(c_{1}, c_{2}, \ldots, \xi_{i}, \ldots, c_{n}\right)$ for all complex $\varepsilon_{i}$-axis.

\section{The growth of a meromorphic function}

This section will provide a brief account of notations and basic facts of value distribution theory of several complex variables that are needed in the proofs of our results. Let $f(\xi)$ be a meromorphic function in $\mathbb{C}^{n} ; d \sigma_{r}$ be the positive 
element of volume on the sphere

$$
S_{n}(r)=\left\{\xi \in \mathbb{C}^{n}|| \xi \mid=r\right\}
$$

Considered as a real $(2 n-1)$-dimensional $C^{\infty}$ manifold, oriented to the exterior of the ball

$$
B_{n}(r)=\left\{\xi \in \mathbb{C}^{n}|| \xi \mid<r\right\}
$$

Also let

$$
V_{n}(r)=\frac{2 \pi^{n} r^{2 n-1}}{(n-1) !}
$$

be the surface area of $S_{n}(r)$ and

$$
\omega_{n}\left(\xi_{1}, \xi_{2}, \ldots, \xi_{n}\right)=\frac{1}{(n-1) !}\left\{\sum_{j=1}^{n} \frac{i}{2} d \xi_{j} \wedge d \bar{\xi}_{j}\right\}^{n-1},
$$

a $C^{\infty}$ complex exterior differential form of bidegree $(n-1, n-1)$ on $\mathbb{C}^{n}$.

Then the characteristic of $f$ is defined by

$$
T_{f}(r, s)=\int_{s}^{r} \frac{A_{f}(t)}{t} d t \quad \text { for } r \geqslant s>0
$$

where

$$
A_{f}(t):=\frac{(n-1) !}{\pi^{n} t^{2 n-2}} \int_{B_{n}(t)} \frac{i}{2\left(1+|f|^{2}\right)^{2}} d f \wedge d \vec{f} \wedge \omega_{n} .
$$

Since $A_{f}(t)$ is a nonnegative, increasing, continuous function on $\mathbb{R}^{+}:=[0,+\infty)$ (see e.g. $[\mathbf{K}, \mathbf{S}]), T_{f}(\cdot, s)$ is a nonnegative increasing $C^{1}$ function on $[s,+\infty)$. The order of $f$ is defined by

$$
\rho(f)=\limsup _{r \rightarrow \infty} \frac{\log ^{+} T_{f}(r, s)}{\log r}, \quad \text { for some } s>0 .
$$

For a meromorphic function $f$ in $\mathbb{C}$, the above characteristic differs from the standard Nevanlinna characteristic $T(r, f)$ by a constant (see e.g. [Ku]). There

$$
T(r, f)=N(r, f)+n(r, f)
$$

with

$$
\begin{aligned}
& N(r, f)=\int_{0}^{r} \frac{n(t, f)-n(0, f)}{t} d t+n(0, r) \log r \\
& m(r, f):=\frac{1}{2 \pi} \int_{0}^{2 \pi} \operatorname{og}^{+}\left|f\left(r e^{i \vartheta}\right)\right| d \theta
\end{aligned}
$$

and $n(r, f):=$ number of poles of $f$ in the disc $|z| \leqslant r$ (see e.g. $[\mathbf{H}]$ ).

For a function $f \neq 0$ holomorphic on an open connected neighborhood of $\xi$ in $\mathbb{C}^{n}$, we have the following series

$$
f(\zeta)==\sum_{j=\nu}^{\infty} P_{i}(\zeta-\xi)
$$


which converges uniformly on some neighborhood of $\xi$ and represents $f$ on this neighborhood. Here $P_{i}$ is a homogeneous polynomial of degree $i$ and $P_{\nu} \not \equiv 0$. The nonnegative integer $\nu$, uniquely determined by $f$ and $\xi$, is called the zero-divisor of $f$ at $\xi$ and is denoted by $\operatorname{div}_{f}(\xi)$ or $\operatorname{div}_{f}^{0}(\xi)$.

If $f$ is a non-constant meromorphic function in $\mathbb{C}^{n}$, then for each $\xi \in \mathbb{C}^{n}$ there exist an open connected neighborhood $U_{\xi}$ of $\xi$ in $\mathbb{C}^{n}$ and two holomorphic functions $g \not \equiv 0$ and $h \not \equiv 0$ on $U_{\xi}$, coprime at $\xi$ (i.e., the germs of $g$ and $h$ are coprime in the local ring of germs of holomorphic functions at $\xi)$, such that $h f=$ $g$ on $U_{\xi}$. The nonnegative integers $\operatorname{div}_{f}^{0}(\xi):=\operatorname{div}_{g}(\xi)$ and $\operatorname{div}_{f}^{\infty}(\xi):=\operatorname{div}_{h}(\xi)$, uniquely determined by $f$ and $\xi$, are called respectively, the zero-divisor and the pole-divisor of $f$. $\operatorname{div}_{f}(\xi):=\operatorname{div}_{f}^{0}(\xi)-\operatorname{div}_{f}^{\infty}(\xi)$ is called the divisor of $f$. $\operatorname{div}_{f}^{\alpha}(\xi):=\operatorname{div}_{g-\alpha h}(\xi)$ is called the $\alpha$-divisor of $f$ if $\alpha \in \mathbb{C}$. Clearly, the function $f$ is holomorphic on $\mathbb{C}^{n}$ if and only if $\operatorname{div}_{f}(\xi) \geqslant 0$ on $\mathbb{C}^{n}$ and $f$ is holomorphic without zeros on $\mathbb{C}^{n}$ if and only if $\operatorname{div}_{f}(\xi) \equiv 0$ on $\mathbb{C}^{n}$.

If $f$ is an entire function in $\mathbb{C}^{n}$ and $\operatorname{div}_{f} \equiv \operatorname{div}_{P}$ for a polynomial $P$ in $\mathbb{C}^{n}$, then $\operatorname{div}_{f}$ is called an algebraic divisor and $Z(f):=\left\{\xi \in \mathbb{C}^{n} \mid f(\xi)=0\right\}$ is called an algebraic variety. Otherwise, $Z(f)$ is said to be transcendental. For an algebraic variety $Z(f)$, if $Z(f)=Z(P)$ for a polynomial $P$ of degree $\leqslant 1$ in $\mathbb{C}^{n}$, then $Z(f)$ is called an algebraic variety of degree $\leqslant 1$, If such a polynomial $P$ does not exist, $Z(f)$ is called an algebraic variety of degree higher than 1. For any meromorphic function $f$ in $\mathbb{C}^{n}$, there are two entire functions $g$ and $h$ such that $h f=g$ on $\mathbb{C}^{n}$ and $g$ and $h$ are coprime at every point of $\mathbb{C}^{n}$ (see e.g. [Ho]). Then $Z(f)$ is called algebraic if $Z(g)$ is algebraic and $P(f):=Z(h)$ is called algebraic if $Z(h)$ is algebraic. We also use the notation $Z(f, g):=\left\{\xi \in \mathbb{C}^{n} \mid f(\xi)=g(\xi)=0\right\}$ for any meromorphic functions $f$ and $g$ in $\mathbb{C}^{n}$, and the notation $D_{u} f:=\frac{\partial f}{\partial \xi_{1}} u_{1}+\cdots+\frac{\partial f}{\partial \xi_{n}} u_{n}$, the directional derivative of $f$ along a unit vector $u \in S^{2 r-1}:=S_{n}(1)$. Now define the differential form

$$
\eta=\frac{1}{4 \pi} d d^{c}|\xi|^{2}
$$

where $d=\partial+\bar{\partial}$ and $d^{c}=\frac{\partial-\bar{\partial}}{i}$. For a meromorphic function $f$ in $\mathbb{C}^{n}$ and $\alpha \in \mathbb{P}^{1}$, the counting function of the $\alpha$-divisor of $f$ is defined by

$$
n_{f}^{\alpha}(r)=r^{2-2 n} \int_{|\xi| \leqslant r, \xi \in \operatorname{supp}\left(\operatorname{div}_{f}^{\alpha}\right)} \operatorname{div}_{f}^{\alpha} \eta^{n-1}
$$

for $n>1$ and

$$
n_{f}^{\alpha}(r)=\sum_{|z| \leqslant r} \operatorname{div}_{f}^{\alpha x}(z)
$$

for $n=1$. The valence function of the $\alpha$-divisor is defined by

$$
N_{f}^{\alpha}(r, s)=\int_{r}^{s} n_{f}^{\alpha}(t) \frac{d t}{t}, \quad \text { for } r \geqslant s>0
$$


The compensation function of $f$ for $\alpha \in \mathbb{P}^{1}$ is defined by

$$
m_{f}^{\alpha}(r)=\frac{1}{V_{n}(r)} \int_{S_{n}(r)} \log \frac{1}{\|f, \alpha\|} d \sigma_{r}
$$

where $\|f, \alpha\|$ is the chordal distance between $f$ and $\alpha$ on the Riemann surface $\mathbb{P}^{1}$.

One has the following first main theorem (see e.g. $[\mathbf{V}, \mathbf{S}]$ ):

$$
T_{f}(r, s)=N_{f}^{\alpha}(r, s)+m_{f}^{\alpha}(r)-m_{f}^{\alpha}(s)
$$

for any $\alpha \in \mathbb{P}^{1}$. This implies that

$$
0 \leqslant \delta_{f}(\alpha):=\liminf _{r \rightarrow \infty} \frac{m_{f}^{\alpha}(r)}{T_{f}(r, s)}=1-\limsup _{r \rightarrow \infty} \frac{N_{f}^{\alpha}(r, s)}{T_{f}(r, s)} \leqslant 1,
$$

where $\delta_{f}(\alpha)$ is called the defect of $f$ for $\alpha$.

\section{Criteria for the primeness of meromorphic functions}

THEOREM 3.1. Let $F(\xi)$ be an entire function of finite order in $\mathbb{C}^{n}$ and $u \in$ $S^{2 n-1}$ be a unit vector. If $Z\left(D_{u} F\right)$ is a transcendental variety and for any $a \in \mathbb{C}$, $Z\left(F-a, D_{u} F\right)$ does not contain any transcendental variety or polynomial variety of degree higher than 1 . Then $F$ is prime.

Proof. First we prove that $F$ is pseudo-prime. Assume, to the contrary, that $F(\xi)=f(g(\xi))$, where $g: \mathbb{C}^{n} \rightarrow \mathbb{C}$ is a transcendental entire function and $f: \mathbb{C} \rightarrow \mathbb{P}^{1}$ is a transcendental meromorphic function.

We consider the restriction $F_{\zeta}(z): \mathbb{C} \rightarrow \mathbb{C}$ of $F^{\prime}(\xi)$ to $\zeta \in S^{2 n-1}$ defined by $F_{\zeta}(z):=F(\zeta z)$. By [Ku Th. 3.9], for any meromorphic function $G$ in $\mathbb{C}^{n}$ with $\operatorname{div}_{G}(0)=0$ and $s>0$ there are constants $A, B$ and $C$ in $\mathbb{R}^{+}$such that $T_{G_{\xi}}(r, s) \leqslant A T_{G}(B r, s)+C$ for $r>0$ and $\xi \in S^{2 n-1}$. Therefore one can deduce immediately, for $\zeta \in S^{2 n-1}$,

$$
\limsup _{r \rightarrow \infty} \frac{\log ^{+} T\left(r, F_{\zeta}\right)}{\log r}<+\infty
$$

by the assumption that $F$ is of finite order. That is, each restriction $F_{\zeta}$ to $\zeta \in S^{2 n-1}$ is of finite order. Since $g(\xi)$ is transcendental, we have the following series expansion

$$
g(\xi)=\sum_{j \geqslant 0}^{\infty} P_{j}(\xi)
$$

where $P_{j}$ is a homogeneous polynomial of degree $j$ and there are infinitely many $j$ 's such that $P_{j} \not \equiv 0$. Denote by $J$ the set of such $j$ 's. For $j \in J$ the set

$$
Z_{j}:=\left\{\xi \in \mathbb{C}^{n} \mid P_{j}(\xi)=0\right\}
$$


is a thin variety and so has $2 n$-dim zero measure (see e.g. [Ra]). Therefore $V:=\cup_{j \in J} Z_{j}$ also has $2 n$-dim zero measure. Take $\zeta \in \mathbb{C}^{n}$ such that $\zeta \neq 0$ and $\zeta \notin V$ and denote $\hat{\zeta}=\frac{\zeta}{|\zeta|}$. Then for sucn $j$,

$$
P_{j}(\hat{\zeta})=P_{j}\left(\frac{\zeta}{|\zeta|}\right)=\frac{1}{|\zeta|^{j}} P_{j}(\zeta) \neq 0 .
$$

This implies that $g_{\hat{\zeta}}(z):=g(\hat{\zeta} z)=\sum_{j}^{\infty} z_{0}^{j} P_{j}(\hat{\zeta})$ is a transcendental entire function of $z$ in $\mathbb{C}$. Notice that $F_{\hat{\xi}}(z)=f\left(g_{\hat{\zeta}}(z)\right)$ and recall that $F_{\hat{\xi}}$ is of finite order. We obtain that $f$ must be of order zero by the theorem in [EF]: if $f$ is a meromorphic function of positive order in $\mathbb{C}$ and $g$ is a transcendental entire function in $\mathbb{C}$ then $f(g(z))$ is of infinite order. Also, for any meromorphic function $h$ in $\mathbb{C}, h$ and its derivative $h^{\prime}$ have the same order (see e.g. $[\mathbf{H}]$ ). We deduce that $f^{\prime}$ is of order zero. We assert that $f^{\prime}$ has at most one pole. Otherwise, it follows from Picard theorent (see e.g. [H]) for one complex variable that, for some pole $z_{0}$ of $f, g_{\zeta_{0}}(z)=z_{0}$ would have infinitely many roots which are all poles of $F(\zeta)$, a contradiction. Thus by the Hadamard factorization for meromorphic function in $\mathbb{C}$ (see e.g. $[\mathbf{T}]$ ), we see that $f^{\prime}$ must have infinitely many zeros, $\left\{z_{m}\right\}_{m=1}^{\infty}$, say. We claim that for all $m \in \mathbb{N}$ (the set of natural integers) with at most two exceptions, the set $Z\left(g-\ddot{z}_{m}\right)$ is a transcendental variety. In fact, if for some $m, Z\left(g-z_{m}\right)$ is algebraic, then there exists a polynomial $P_{m}(\zeta)$ in $\mathbb{C}^{n}$ such that $Z\left(g-z_{m}\right)=Z\left(P_{m}\right)$. Remember for any polynomial $P(\zeta)$ in $\mathbb{C}^{n}, T_{P}(r, s)=O(\log r)$ and for any transcendental meromorphic function $h(\xi)$ in $\mathbb{C}^{n}, \log r=o\left\{T_{h}(r, s)\right\}$ as $r \rightarrow \infty$ (see e.g. [G, S]). Therefore $N_{g}^{z_{m}}(r, s)=$ $N_{P_{m}}^{0}(r, s) \leqslant T_{P_{m}}(r, s)=O(\log r)$ and

$$
\log r=o\left\{T_{g}(r, s)\right\}
$$

as $r \rightarrow \infty$. This implies that the defect

$$
\delta_{g}\left(z_{m}\right):=1-\limsup _{r \rightarrow \infty} \frac{N_{g}^{z_{m}}(r, s)}{T_{g}(r, s)}=1 .
$$

However, by the defect relation (see [V] r [S]) for meromorphic functions in $\mathbb{C}^{n}$,

$$
\sum_{m=1}^{\infty} \delta_{g}\left(z_{m}\right) \leqslant 2
$$

Therefore, the above claim holds. On the other hand, it is easy to see that $Z\left(g-z_{n}\right) \subset Z\left(F-f\left(z_{n}\right), D_{u} F\right)$. We arrive at a contradiction to the hypothesis that for any $a \in \mathbb{C}, Z\left(F-a, D_{u} F\right)$ does not contain any transcendental variety. We have thus proved the pseudo-primeress of $F$.

Now we prove that $F$ is left-prime. By the above result, we may assume that $F(\xi)=P(g(\xi))$, where $P(z): \mathbb{C} \rightarrow \mathbb{P}^{1}$ is a rational function of order $d \geqslant 1$ and $g(\xi): \mathbb{C}^{n} \rightarrow \mathbb{P}^{1}$ is a meromorphic function. It suffices to prove that $d=1$. We discuss two cases. 
Case 1: $P(\infty)=\infty$. In this case $g(\xi)$ mus be an entire function in $\mathbb{C}^{n}$ since otherwise $F(\xi)$ would have poles. We assert that $P^{\prime}(z)$ cannot have any zeros in $\mathbb{C}$. If, to the contrary, $P^{\prime}\left(z_{0}\right)=0$ for some $z_{0} \in \mathbb{C}$, then $\operatorname{div}_{g-z_{0}}$ must be an algebraic divisor since otherwise we would have that the transcendental variety $Z\left(g-z_{0}\right)$ belongs to $Z\left(F-P\left(z_{0}\right), D_{u} F\right)$, a contradiction to the hypothesis of the theorem. Thus we can find a polynomial $R(\xi)$ in $\mathbb{C}^{n}$ such that $\operatorname{div}_{g-z_{0}}=\operatorname{div}_{R}$ in $\mathbb{C}^{n}$. This means that $\frac{g(\xi)-z_{0}}{R(\xi)}$ is an entire function without any zeros in $\mathbb{C}^{n}$. We then have that

$$
g(\xi)-z_{0}=R(\xi) e^{S(\xi)}
$$

where $S(\xi)$ is an entire function in $\mathbb{C}^{n}$. For any $\zeta \in S^{2 n-1}, F_{\zeta}(Z)=P\left(g_{\zeta}(Z)\right)$. Since $F$ is of finite order, its restriction $F_{\zeta}$ to $\zeta$ must be of finite order (see the above proof of the pseudo-primeness). But by [BJV], for any meromorphic function $h(z)$ and rational function $Q(z)$ of degree $q$ in $\mathbb{C}, T(r, Q(h))=q T(r, h)+$ $O(\log r)$ as $r \rightarrow \infty$. Therefore $g_{\zeta}(z)$ is of finite order for each $\zeta \in S^{2 n-1}$. By (3.1), $g_{\zeta}(z)-z_{0}=R_{\zeta}(z) e^{S_{\zeta}(z)}$. Hence for each $\zeta \in \mathbb{C}^{n}$ with $\zeta \notin Z(R)$, which is a thin variety, we must have that $S_{\xi}(Z)$ is a polynomial in $\mathbb{C}$, where $\hat{\zeta}=\frac{\zeta}{|\zeta|}$. This shows that in $(3.1), S(\xi)$ turns out to be a polynomial in $\mathbb{C}^{n}$. Otherwise, by the same proof as in the pseudo-primeness, for all $\zeta \in \mathbb{C}^{n}$ except for a set of $2 n$-dim zero measure, $S_{\bar{\zeta}}(Z)$ is transcendental, a contradiction to the above. Obviously, by $(3.1)$

$$
D_{u} g(\xi)=\left(D_{u} K(\xi)+R(\xi) D_{u} S(\xi)\right) e^{S(\xi)} .
$$

Since $R(\xi)$ and $S(\xi)$ are all polynomials in $\mathbb{C}^{n}$, we see that

$$
\operatorname{div}_{D_{u} g}=\operatorname{div}_{D_{u}} R+R D_{u} S+\operatorname{div}_{e} s=\operatorname{div}_{D_{u}} R+R D_{u} S
$$

an algebraic divisor. By the hypothesis, $Z\left(D_{u} F\right)$ is a transcendental variety and $D_{u} F=P^{\prime}(g) D_{u} g$. Therefore there must exist a $z_{1} \in \mathbb{C}$ such that $P^{\prime}\left(z_{1}\right)=0$ and $Z\left(g-z_{1}\right)$ is a transcendental variety. This is a contradiction since $Z\left(g-z_{1}\right) \subset$ $Z\left(F-P\left(z_{1}\right), D_{u} F\right)$. It thus follows that $P^{\prime}(z)$ cannot have any zeros in $\mathbb{C}$. Set

$$
P(z)=A \frac{q_{1}(z)}{q_{2}(z)}=A \frac{\left(z-a_{1}\right)^{n_{1}}\left(z-a_{2}\right)^{n_{2}} \cdots\left(z-a_{N}\right)^{n_{N}}}{\left(z-b_{1}\right)^{m_{1}}\left(z-b_{2}\right)^{m_{2}} \cdots\left(z-b_{M}\right)^{m_{M}}},
$$

where $A$ is a non-zero constant, $q_{1}(z)$ and $q_{2}(z)$ are two coprime polynomials in $\mathbb{C}$. Since $P(\infty)=\infty, d=\sum_{j=1}^{N} n_{j}>e:=\sum_{j=1}^{M} m_{j} \geqslant 0$. It is easy to see that

$$
P^{\prime}(z)=A \frac{q_{1}^{\prime}(z) \prod_{j=1}^{M}\left(z-b_{j}\right)-q_{1}(z) \sum_{j=1}^{M}\left(m_{j} \prod_{i=1, i \neq j}^{M}\left(z-b_{i}\right)\right)}{\prod_{j=1}^{M}\left(z-b_{j}\right)^{m_{j}+1}}
$$




$$
=A \frac{q(z)}{\prod_{j=1}^{M}\left(z-b_{j}\right)^{m_{j}+1}}
$$

with the obvious definition of $q(z)$. Since $q_{1}\left(b_{j}\right) \neq 0$ for $1 \leqslant j \leqslant M, q\left(b_{j}\right) \neq 0$ for $1 \leqslant j \leqslant M$. Thus $q(z)$ and $\prod_{j=1}^{M}\left(z-b_{i}\right)^{m_{j}+1}$ are coprime. However the leading term of $q(z)$ is $d z^{d-1+M}-\left(m_{1}+m_{2}+\cdots+m_{M}\right) z^{d+M-1}=(d-e) z^{d+M-1}$. Recall that $P^{\prime}(z)$ and so $q(z)$ do not have any zero in $\mathbb{C}$. We thus deduce that $d+M-1=0$ and so that $d=1$ and $\epsilon=0$ by the fact that $d>e \geqslant M \geqslant 0$.

Case 2: $P(\infty) \neq \infty$. In this case, $F^{\prime}(z)$ must have a pole, $w_{0}$ say. Hence

$$
P(z)=\frac{1}{\left(z-w_{0}\right)^{m}} q_{3}(z)=\left\{w^{m} q_{3}\left(w_{0}+\frac{1}{w}\right)\right\}:=q_{4}(w)
$$

where $m$ is a positive integer, $q_{3}(z)$ is a rational function in $\mathbb{C}$ with $q_{3}\left(w_{0}\right) \neq 0$, and $w=\frac{1}{z-w_{0}}$ is a Möbius transformation. Clearly $q_{4}(w)$ is a rational function of $w$ and $q_{4}(\infty)=\infty$. Notice that

$$
F(\xi)=P(g(\xi))==q_{4}\left(\frac{1}{g(\xi)-w_{0}}\right) .
$$

By the result of case (1), we must have that

$$
q_{4}(w)==a w+b
$$

for some constants $a, b$ with $a \neq 0$ and so that

$$
P(z)=q_{4}(w)=q_{4}\left(\frac{1}{z-w_{0}}\right)=a \frac{1}{z-w_{0}}+b
$$

is a rational function of degree 1 , i.e. $d=1$. This completes the proof of the left-primeness of $F$.

Next, it remains to prove the right-primeness. By the pseudo-primeness of $F$, we may assume that

$$
F(\xi)=f(Q(\xi))
$$

where $f$ is a transcendental meromorphic function from $\mathbb{C}$ to $\mathbb{P}^{1}$ and $Q(\xi)$ is a polynomial of degree $\rho(\geqslant 1)$ in $\mathbb{C}^{n}$. Clearly

$$
D_{u} F=f^{\prime}(Q) D_{\imath} Q \text {. }
$$

By the hypothesis that $Z\left(D_{u} F\right)$ is transcendental, $f^{\prime}(z)$ must have infinitely many zeros, $\left\{a_{m}\right\}_{m=1}^{\infty}$, say. Since for any $m \in \mathbb{N}$,

$$
Z\left(Q-a_{m}\right) \subset Z\left(F-f\left(a_{m}\right), D_{u} F\right)
$$

and $Z\left(F-f\left(a_{m}\right), D_{u} F\right)$ does not contain any transcendental variety and algebraic variety of degree higher than 1 by the hypothesis, we deduce that

$$
Z\left(Q-a_{m}\right)=Z\left(Q_{m}\right)
$$


for a polynomial $Q_{m}$ of degree $\leqslant 1$ in $\mathbb{C}^{n}$. By writing $Q-a_{m}$ into the product of irreducible functions, it is easy to see that $q-a_{m}$ must assume the following form

$$
Q-a_{m}=\left(c_{m}^{(0)}+c_{m}^{(1)} \xi_{1}+c_{m}^{(2)} \xi_{2}+\cdots+c_{m}^{(n)} \xi_{n}\right)^{\rho}
$$

where $c_{m}^{(0)}, \ldots, c_{m}^{(n)}$ are constants and $\xi_{j}$ is the $j$-th coordinate of $\xi$. Also we can write $Q(\xi)$ into a Weierstrass pseudo polynomial in some variable, $\xi_{1}$ say, such that, in view of $(3.2)$,

$$
Q(\xi)=Q\left(\xi_{1}, \xi_{2}, \ldots, \xi_{n}\right)=\alpha_{\rho} \xi_{1}^{\rho}+\alpha_{\rho-1}\left(\xi_{2}, \ldots, \xi_{n}\right) \xi_{1}^{\rho-1}+\cdots+\alpha_{0}\left(\xi_{2}, \ldots, \xi_{n}\right)
$$

where $\alpha_{\rho} \neq 0$ is a constant, and $\alpha_{\rho-1}, \ldots, \alpha_{0}$ are polynomials of $\xi_{2}, \ldots, \xi_{n}$. Therefore by letting $\xi_{2}=0, \xi_{3}=0, \ldots, \xi_{n}=0$, we get a polynomial $Q^{*}\left(\xi_{1}\right):=$ $Q\left(\xi_{1}, 0,0, \ldots, 0\right)$ of degree $\rho$ in $\xi_{1}$. It follows that for large $m, Q^{*}\left(\xi_{1}\right)-a_{m}$ has $\rho$ distinct zeros in the plane. However by $(3.2), Q^{*}\left(\xi_{1}\right)-a_{m}=\left(c_{m}^{(0)}+c_{m}^{(1)} \xi_{1}\right)^{\rho}$ has only one zero of multiplicity $\rho$. Therefore it follows that $\rho=1$. This shows that $F$ is right-prime.

Summarizing the above results, we have showed that $F$ is both left-prime and right-prime. Therefore $F$ must be prime. The proof of the theorem is thus complete.

Observing that in the proof of the left-primeness of Theorem 3.1, we did not use the condition that " $Z\left(F-a, D_{u} F\right)$ does not contain any polynomial variety of degree higher than 1 ", we thus have the following proposition for the left-primeness.

Proposition 3.2. Let $F(\xi)$ and $u \in S^{2 n-1}$ be the same as in Theorem 3.1. If $Z\left(D_{u} F\right)$ is a transcendental variety and for any $a \in \mathbb{C}, Z\left(F-a, D_{u} F\right)$ does not contain any transcendental variety, then $F$ is left-prime.

REMARK 3.3. It is natural to ask whether or not $F$ is prime under the hypotheses of proposition 3.2. A negative answer is given by the following counter example. Consider, in $\mathbb{C}^{n}$, the function

$$
F(\xi)=\cos \left(\xi_{1}+\xi_{2}+\cdots+\xi_{n}\right)+\left(\xi_{1}+\xi_{2}+\cdots+\xi_{n}\right)^{2}
$$

Clearly, $F(\xi)$ is an entire function of finite order. Let.

$$
u=(1,0, \ldots, 0) \text {. }
$$

Then

$$
D_{u} F=\frac{\partial F}{\partial \xi_{1}}=-\sin \left(\xi_{1}+\xi_{2}+\cdots+\xi_{n}\right)+2\left(\xi_{1}+\xi_{2}+\cdots+\xi_{n}\right) .
$$

We assert that $Z\left(D_{u} F\right)$ is a transcendental variety. Otherwise there would be two polynomials $P_{1}(\xi)$ and $P_{2}(\xi)$ in $\mathbb{C}^{n}$ such that

$$
D_{u} F=P_{1}(\xi) e^{P_{2}(\xi)}
$$


and so that

$$
\begin{aligned}
D_{u}^{(3)} F: & =D_{u}\left(D_{u}\left(D_{u} F\right)\right) \\
& =\left\{\frac{\partial}{\partial \xi_{1}}\left(\frac{\partial P_{1}}{\partial \xi_{1}}+P_{1} \frac{\partial P_{2}}{\partial \xi_{i}}\right)+\left(\frac{\partial P_{1}}{\partial \xi_{1}}+P_{1} \frac{\partial P_{2}}{\partial \xi_{1}}\right) \frac{\partial P_{2}}{\partial \xi_{1}}\right\} e^{P_{2}} .
\end{aligned}
$$

This means that $\operatorname{div}_{D_{u}^{(3)} F}$ is a polynomial divisor. However by (3.3),

$$
\operatorname{div}_{D_{u}^{(3)} F}=\sin \left(\xi_{1}+\xi_{2}+\cdots+\xi_{n}\right)
$$

and thus $\operatorname{div}_{D_{u}^{(3)} F}=\operatorname{div}_{\sin \left(\xi_{1}+\xi_{2}+\cdots+\xi_{n}\right)}$, a non-polynomial divisor. We obtain a contradiction. Next, for any $a \in \mathbb{C}$, it is easy to check that

$$
Z\left(F-a, D_{u} F\right) \subset Z(Q(Z))
$$

where $Q(Z):=\left\{a-\left(\xi_{1}+\cdots+\xi_{n}\right)^{2}\right\}^{2}+4\left(\xi_{1}+\cdots+\xi_{n}\right)-1$ is a polynomial in $\mathbb{C}^{n}$. Thus $Z\left(F-a, D_{u} F\right)$ does not contain any transcendental variety. But

$$
F(Z)^{*}=(\cos \sqrt{Z}+Z) \circ\left(\xi_{1}+\cdots+\xi_{n}\right)^{2}
$$

is not prime in $\mathbb{C}^{n}$. This example shows that in Theorem 3.1, one cannot drop the condition: " $Z\left(F-a, D_{u} F\right)$ does not contain any polynomial variety of degree higher than 1".

COROLlaRY 3.4. $F(\xi)=e^{\left(\xi_{1}+\xi_{2}+\cdots+\xi_{n}\right)}+a_{1} \xi_{1}+a_{2} \xi_{2}+\cdots+a_{n} \xi_{n}$ is prime, where $a_{i}$ 's are complex numbers not all zero. Then

PRoOF. It is no loss of generality to assume that $a_{1} \neq 0$. Let $u=(1,0, \ldots, 0)$.

$$
D_{u} F=e^{\xi_{1}+\xi_{2}+\cdots+\xi_{n}}+a_{1} .
$$

Clearly $F$ is of finite order, $Z\left(D_{u} F\right)$ is a transcendental variety and for any $a \in \mathbb{C}_{\text {, }}$

$$
Z\left(D_{u} F, F-a\right)=Z(Q(\xi)),
$$

where $Q(\xi)=a_{1} \xi_{1}+a_{2} \xi_{2}+\cdots+a_{n} \xi_{n}-\left(a-a_{1}\right)$ is a polynomial of degree 1 . By Theorem $3.1, F(Z)$ is prime.

There are a large number of functions satisfying the hypotheses of Theorem 3.1 and thus being prime, for example, it is easy to verify that $F_{1}(\xi)=\sin \left(\xi_{1}+\right.$ $\left.\xi_{2}+\cdots+\xi_{n}\right)+a_{1} \xi_{1}+\cdots+a_{n} \xi_{n}$ and $F_{2}(\xi)=\cos \left(\xi_{1}+\xi_{2}+\cdots+\xi_{n}\right)+a_{1} \xi_{1}+\cdots+a_{n} \xi_{n}$ are all prime, where $a_{i}$ 's are complex numbers not all zero.

Letting $n=1$ in proposition 3.2 and Theorem 3.1 , we have the following result for the factorization of one complex variable, which is interesting in itself.

COROLlaRY 3.5. Let $F^{\prime}(z)$ be an entire function of finite order in the complex plane. If $F^{\prime}(z)$ has infinitely many zeros and for any $a \in \mathbb{C}, Z\left(F-a, F^{\prime}\right)$ contains finitely many points, then $F$ is left-prime. Moreover, if $Z\left(F-a, F^{\prime}\right)$ contains at most one point, then $F$ is prime.

REMARK 3.6. As pointed out in Section $1, F(z)=e^{z}+z$ is a fundamental prime function in $\mathbb{C}$. Several different proofs have been found in the literature. 
Now the primeness of this function is a direct consequence of Corollary 3.4 or Corollary 3.5. We also remark here that for an entire function $F(z)$, one can define the concepts of E-prime, E-pseudo-prime, E-left-prime and E-right-prime. $F$ is called E-prime (E-pseudo-prime, E-left-prime, E-right-prime) if $F$ is prime (pseudo-prime, left-prime, right-prime) when only entire factors $f$ and $g$ are considered in the factorization of form (1.1) or (1.2). Obviously, that $F$ is prime (pseudo-prime, left-prime, right-prime) implies that $F$ is E-prime (E-pseudoprime, E-left-prime, E-right-prime). But the converse is not true even in the case when $n=1$ (For example, the function $e^{-m z}+e^{-n z}$ is E-prime but not prime, where $m, n \in \mathbb{N}$ ). As a direct consequence of Corollary 3.5 , we also have the corresponding criteria for the E-primeness of entire functions in $\mathbb{C}$. In particular, one obtains Ozawa's result: under the hypotheses of Corollary 3.5, F is E-left-prime (see [O]).

we conclude this section by pointing out that in Theorem 3.1, the function $F$ can also be meromorphic in $\mathbb{C}^{n}$ provided that $P\left(F^{\prime}\right)$ is algebraic. That is, we have the following

THEOREM 3.7. Let $F(\xi)$ be a meromorphic function of finite order in $\mathbb{C}^{n}$ and $u \in S^{2 n-1}$ be a unit vector. If $P(F)$ is an algebraic variety, $Z\left(D_{u} F\right)$ is a transcendental variety and for any $a \in \mathbb{C}, Z\left(F-a, D_{u} F\right)$ does not contain any transcendental variety or polynomial variety of degree higher than 1 . Then $F$ is prime.

The proo $f$ is a simple modification of the one of Theorem 3.1. We thus omit the details here.

\section{The comparison between the primeness} of $F(\xi)$ and $F\left(c_{1}, c_{2}, \ldots, \xi_{i}, \ldots, c_{n}\right)$

Let $F(\xi)$ be a meromorphic function in $\mathbb{C}^{n}$. We consider the relation between the primeness of $F(\xi)$ and its restriction $F\left(c_{1}, c_{2}, \ldots, \xi_{i}, \ldots, c_{n}\right)$ to each complex $\xi_{i}$-axis. It is natural to ask whether or not the primeness of $F(\xi)$ is equivalent to the primeness of $F\left(c_{1}, c_{2}, \ldots, \xi_{i}, \ldots, c_{n}\right)$ for $1 \leqslant i \leqslant n$ and any fixed constants $c_{j}(1 \leqslant j \leqslant n, j \neq i)$. The answer is negative.

Let us consider the entire function

$$
F\left(\xi_{1}, \xi_{2}, \ldots, \xi_{n}\right)=\left(1+\prod_{j=1}^{n} \xi_{j}\right) e^{\Pi_{j=1}^{n} \xi_{j}}+\prod_{j=1}^{n} \xi_{j}
$$

in $\mathbb{C}^{n}$. Clearly

$$
F\left(\xi_{1}, \xi_{2}, \ldots, \xi_{n}\right)=\left((1+z) e^{z}+z\right) \circ\left(\prod_{j=1}^{n} \xi_{j}\right)
$$

is not prime in $\mathbb{C}^{n}$. However, for any $1 \leqslant i \leqslant n$ and fixed constants $c_{j}(1 \leqslant j \leqslant n$, 
$j \neq i)$

$$
\begin{aligned}
F\left(c_{1}, c_{2}, \ldots, \xi_{i}, \ldots, c_{n}\right) & =\left(1+\left(\prod_{j=1, j \neq i}^{n} c_{j}\right) \xi_{2}\right) e^{\left(\prod_{j=1, j \neq i}^{n} c_{j}\right) \xi_{i}}+\left(\prod_{j=1, j \neq i}^{n} c_{j}\right) \xi_{i} \\
& =\left(1+c \xi_{i}\right) e^{c \xi_{i}}+c \xi_{i} \quad\left(c:=\prod_{j=1, j \neq i}^{n} c_{j}\right)
\end{aligned}
$$

is a prime function of $\xi_{i}$ (see e.g. [G]) This example shows that the primeness of $F\left(c_{1}, c_{2}, \ldots, \xi_{i}, \ldots, c_{n}\right)$ for $1 \leqslant i \leqslant n$ does not guarantee that $F(\xi)$ is prime in $\mathbb{C}^{n}$

In the opposite direction, the primeness of $F(\xi)$ also does not guarantee $F\left(c_{1}, c_{2}, \ldots, \xi_{i}, \ldots, c_{n}\right)$ is prime as shown by the following counter example. Consider the function

$$
F(\xi)=F^{\prime}\left(\xi_{1}, \xi_{2}, \ldots, \xi_{n}\right)=e^{\sum_{j=1}^{n} \xi_{j}}+\xi_{1} .
$$

By Corollary3.4, $F(\xi)$ is prime in $\mathbb{C}^{n}$. However for any fixed constants $c_{j}(1 \leqslant$ $j \leqslant n-1), F\left(c_{1}, c_{2}, \ldots, c_{n-1}, \xi_{n}\right)$, the restriction of $F(\xi)$ to the variable $\xi_{n}$, is not prime in $\xi_{n}$ since

$$
F\left(c_{1}, c_{2}, \ldots, c_{n-1}, \xi_{n}\right)=e^{\sum_{j=1}^{n-1} c_{j}+\xi_{n}}+c_{1}=f(g),
$$

where $f(Z)=Z^{2}+c_{1}$ and $g\left(\xi_{n}\right)=e^{\frac{1}{2}\left(\sum_{j=1}^{n-1} c_{j}+\xi_{n}\right)}$.

In the positive direction, we have the following theorem which shows that by adding some extra conditions one can get the primeness of $F(\xi)$ from the primeness of all restrictions of $F(\xi)$. For the sake of simpleness, we restrict to two complex variables.

THEOREM 4.1. Let $F(\xi)$ be a meromorphic function in $\mathbb{C}^{2}$. If $F(c, z), F(z, c)$ and $F(s z, t z)$ are all prime in $\mathbb{C}$, where $c, s, t$ are any fixed complex numbers with st $\neq 0$, then $F(\xi)$ is prime ir. $\mathbb{C}^{2}$.

Proof. Let $F(\xi)=f(g(\xi))$ be a factorization of form (1.2). Then

$$
F(c, z)=f(g(c, z)) \text { and } F(z, c)=f(g(z, c)) .
$$

Since $F(c, z)$ is prime, we have that either $f$ is bilinear or $g$ is linear. If $f$ is bilinear, then there is nothing to prove. We may assume that $f$ is not linear. Then $g(c, z)$ and $g(z, c)$ must be linear. We thus have

$$
g\left(c, \xi_{2}\right)=a_{1}(c) \xi_{2}+a_{2}(c)
$$

and

$$
g\left(\xi_{1}, c\right)=b_{1}(c) \xi_{1}+b_{2}(c)
$$

where $a_{1}, a_{2}, b_{1}, b_{2}$ are meromorphic functions in $\mathbb{C}$.

It follows that

$$
g\left(\xi_{1}, \xi_{2}\right)=a_{1}\left(\xi_{1}\right) \xi_{2}+a_{2}\left(\xi_{1}\right)=b_{1}\left(\xi_{2}\right) \xi_{1}+b_{2}\left(\xi_{2}\right) .
$$


In (4.1), letting $\xi_{2}=0$, we have

$$
a_{2}\left(\xi_{1}\right)=b_{1}(0) \xi_{1}+b_{2}(0)
$$

and letting $\xi_{2}=1$, we have

$$
a_{1}\left(\xi_{1}\right)+a_{2}\left(\xi_{1}\right)=b_{1}(1) \xi_{1}+b_{2}(1) .
$$

Therefore,

$$
\begin{aligned}
a_{1}\left(\xi_{1}\right) & =b_{1}(1) \xi_{1}+b_{2}(1)-a_{2}\left(\xi_{1}\right) \\
& =b_{1}(1) \xi_{1}+b_{2}(1)-b_{1}(0) \xi_{1}-b_{2}(0):=a \xi_{1}+b,
\end{aligned}
$$

where $a=b_{1}(1)-b_{1}(0)$ and $b=b_{2}(1)-b_{2}(0)$. Then by (4.2),

$$
\begin{aligned}
a_{2}\left(\xi_{1}\right) & =b_{1}(1) \xi_{1}+b_{2}(1)-a_{1}\left(\xi_{1}\right) \\
& =b_{1}(1) \xi_{1}+b_{2}(1)-a \xi-b:=c \xi_{1}+d
\end{aligned}
$$

where $c=b_{1}(1)-a$ and $d=b_{2}(1)-b$. Now (4.1) yields that

$$
\begin{aligned}
g\left(\xi_{1}, \xi_{2}\right) & =\left(a \xi_{1}+b\right) \xi_{2}+c \xi_{1}+d \\
& =a \xi_{1} \xi_{2}+b \xi_{2}+c \xi_{1}+d .
\end{aligned}
$$

On the other hand, $F(s z, t z)$ is a prime function of $z$. Hence $g(s z, t z)$ must be linear. This implies that $a=0$ in (4.3). We then obtain that $g\left(\xi_{1}, \xi_{2}\right)=$ $b \xi_{2}+c \xi_{1}+d$ is a linear function in $C^{2}$.

The proof of Theorem 4.1 is thus complete.

Theorem 4.1 enables us to decide whether or not some functions are prime in $\mathbb{C}^{n}$ by using known results in $\mathbb{C}$. For example, one can immediately see that $e^{\xi_{1}}+e^{\xi_{2}}+\xi_{1}+\xi_{2}$ is a prime function in $\mathbb{C}^{2}$.

\section{REFERENCES}

[BG] I. N. Baker and F. Gross, Further results on factorization of entire functions, Entire Functions and Related Parts of Analysis, (Proc. Symp. Pure Math., La Jolla, CA 1966), American Mathematical Society, Providence, RI, 1968, pp. 30-35.

[BJV] W. Bergweiler, G. Jank and L. Volkman, Wachstumsverhalten Zusammengesetzer Funktionen, Result in Math. 7 (1984), 35-53.

[CY] C. T. Chuang and C. C. Yang, Theory of Fix Points and Factorization of Meromorphic Functions, Mathematical Monograph Series, Peking University Press, 1986.

[EF] A. Edrie and W. H. J. Fucks, $O n$ the zeros of $f(g(z))$ where $f$ and $g$ are entire functions, J. Analyse Math. 12 (1964), 243-255.

[G] P. A. Griffiths, Entire holomorphic mapping in one and several variables, Princeton University Press, 1976.

[G1] F. Gross, On factorization of meromorphic functions, Trans. Amer. Math. Soc. 131 (1968), 215-222.

[G2] On factorization of meromorphic functions which are periodic mod $g$, Indian Journ. Pure Appl. Math. 2 (1971), 561-571.

[G3] Factorization of meromorphic functions, U. S. Government Printing Office, Washington D. C., 1972.

[H] W. K. Hayman, Meromorphic Functions, Oxford Math. Monographs, Clarendon Press, Oxford, 1964.

[Ho] L. Hörmander, An Introduction to Complex Analysis in Several Variables, Van Nostrand, Princeton, NJ, 1966. 
[K] H. Kneser, Zur Theorie der gebrochenen Funktionen mehrerer Veränderlichen, Jber. Deutsch Math. Verein 48 (1938), 1-28.

[Ku] R. O. Kujala, Functions of finite $\wedge$-type in several complex variables, Trans. Amer. Math. Soc. 161 (1971), 327-358.

[O] M. Ozawa, On certain criteria for the left-primeness of entire functions, Kodai Math. Sem. Rep. 6 (1975), 304-317.

[Ra] R. M. Range, Holomorphic Functions and Integral Representations in Several Complex Variables, Springer-Verlag, New York, 1986.

[R] P. C. Rosenbloom, The fix-points of entire functions, Medd. Lunds Univ. Mat. Sem. Suppl. Bd. M. Riesz (1952), 186-192.

[S] W. Stoll, Introduction to Value Distribution Theory of Meromorphic Maps, Lecture Notes in Math. No. 950, Springer-Verlag, New York, 1982, pp. 210-359.

[T] E. C. Titchmarsh, The Theory of Furctions, 2nd ed., Oxford Univ. Press, London, 1939.

[V] A. Vitter, The lemma of the logarithmıc derivative in several complex variables, Duke Math. J. 44 (1977), 89-1.04.

[Y1] C. C. Yang, On the dependence of zeros of an entire function and its factorization, J. Math. Anal. Appl. 35 (197:), 374-380.

[Y2] - Some aspects of factorization theory - a survey, Complex Variables 13 (1989), $133-142$.

Department of Mathematics, University of Maryland, College Park, MD 20742 , U. S. A.

Department of Mathematics, The Hong Kong University of Science and TechNOLOGY, KOWLOON, HONG KONG 